\title{
Thyrotoxic Cardiomyopathy
}

\author{
Alina Yu. Babenko, Alekber A. Bairamov, \\ Elena N. Grineva and Eugenia O. Ulupova \\ Almazov Federal Heart, Blood and Endocrinology Centre, St. Petersburg, \\ Russia
}

\section{Introduction}

\subsection{Thyrotoxicosis and thyrotoxic cardiomyopathy - Current state}

Thyrotoxicosis is a common syndrome, and according to the Wickham Survey its prevalence is $2 \%$ among women and $0.2 \%$ in men in the areas with normal iodine consumption. Over the 20-year follow-up the incidence of thyrotoxicosis was 0.8:1000 in women and 0.6:1000 in men (Bird et al,1977).

Subclinical thyrotoxicosis (ST) is defined by low or undetectable serum thyrotropin stimulating hormone (TSH) and normal free thyroxin (FT4) and free triiodthyronin (FT3) concentrations (Biondi \& Cooper, 2008). Although thyroid hormones are defined as being within normal range in patients with ST, they are often at the upper limit of the reference range, and thus sufficiently increased to suppress TSH and potentially produce abnormal tissue effects.

The prevalence of subclinical thyrotoxicosis varies from 0.6 to $16 \%$ \% (Biondi et al., 2002; Wang \& Crapo, 1997; Samuels, 1998) depending on such risk factors as age (it is twice higher in elderly people), iodine deficiency (5-times higher in the areas of iodine deficiency), and decreased TSH level. All authors agree that ST is more common compared to overt thyrotoxicosis.

There are exogenous (iatrogenic) and endogenous causes of thyrotoxicosis. Exogenous thyrotoxicosis usually is subclinical and mainly is caused by suppressive therapy with Lthyroxin leading to the decrease of TSH below normal values. It is commonly present in postoperative patients with differentiated thyroid cancer (papillary and follicular) as the mean of cancer recurrence prevention. This group of patients gets prolonged (2 years and longer) high-dose L-thyroxin therapy, and is considered in studies regarding side-effects of suppressive treatment. At the same time patients with benign thyroid nodules get short courses (6-12 months) of suppressive therapy, leading to a less decrease of TSH (above 0,1 $\mathrm{mIU} / 1$ ). According to the majority of data $1 / 2-2 / 3$ of ST cases are exogenous (Figge et al., 1994; Canaris et al., 2002; Hoogendoorn et al., 2004).

Endogenous thyrotoxicosis can be also divided into immune (Graves' disease, "Hashitox") and non-immune (toxic (pretoxic) thyroid adenoma and toxic multinodular goiter) (Ross, 1996; Marqusee et al., 1998; Al-Abadi, 2001). Graves' disease is the most common cause of overt thyrotoxicosis.

Thyrotoxic cardiomyopathy (TCMP) is a threatening complication of thyrotoxicosis increasing risk of disability and mortality. Until recently myocardial alterations observed in 
thyrotoxicosis were considered favorable regarding prognosis because of the high reversibility if euthyroid state is quickly achieved. However, long-term follow-up studies demonstrated that the involution of the changes is not always complete. Some irreversible histological alterations of myocardium are found in patients who had thyrotoxicosis in past: necrosis of the hypertrophied myocytes and fibrosis development (Ortmann et al., 1999). Myocardial fibrosis has been also detected by radioimmunoimaging with antimyosin antibodies in patients with TCMP complicated by heart failure (HF) (Martí et al., 1997).

There is a current evidence that even successfully treated thyrotoxicosis is associated with the worse prognosis. Thus, cardiovascular (hypertension, valvular diseases, heart failure, and to a lesser extent coronary artery disease) mortality is 1.2 higher in these patients, and cerebrovascular mortality is 1.4 higher than in general population (Osman et al., 2002). Persistent cardiovascular alterations are considered an evident cause of the increased mortality in thyrotoxicosis (Metso et al., 2007; Sheu et al., 2010). Health effects of subclinical thyrotoxicosis are not fully recognized, and some questions are still unsolved. However, quite large amount of publications of the last two decades verify deleterious effects of thyrotoxicosis, and uppermost, on cardiovascular system (Biondi et al., 2002). Moreover, there is evidence of the increased mortality in subclinical thyrotoxicosis (Sgarbi, 2010). Thus, in British study among patients over 60 years of age mortality ( 5 year follow-up) in subjects with TSH below normal values was about twice that of euthyroid subjects (Parle et al., 2001). Cardiovascular diseases were the most common cause of lethal events. Therefore, cardiovascular complications of thyrotoxicosis are of great medical importance.

\section{Definition - Epidemiology of thyrotoxic cardiomyopathy and its clinical variants}

Thyrotoxic cardiomyopathy defines a myocardial damage caused by the toxic effects of abundant thyroid hormones (TH) (Report of the 1995 WHO/ISFC) that result in altered energy production by myocytes (oxidative phosphorylation, glycolysis), intracellular metabolism (protein synthesis) and myofibril contractile function (Klein, 1990).

The main manifestations of thyrotoxic cardiomyopathy (TCMP) are left ventricular hypertrophy (LVH) (Ching et al., 1996, Dorr et al., 2005), heart rhythm disturbances (usually, atrial fibrillation (AF)) (Dunn et al., 1986; Frost et al., 2004), dilation of the heart chambers and heart failure (HF) (Dougherty \& Craige, 1973; Siu et al., 2007), pulmonary hypertension (PH) (Chu et al., 2002; Rubin \& Badesch, 2006), and diastolic dysfunction. Epidemiology data on TCMP are currently lacking. This is due to the lack of diagnostic criteria and to the late introduction of the term "thyrotoxic cardiomyopathy" in clinical and research practice that occurred only after implementation of WHO classification of cardiomyopathies (Report of the 1995 WHO/ISFC).

Meanwhile et al. (1959) found that 4 out of 9 postmortem examinations detected no other cause for severe congestive HF but thyrotoxicosis, and that 64 out of 150 patients (43\%) with thyrotoxicosis had cardiovascular complications in the absence of any known pre-existing heart disease.

The available evidence provides only data on the incidence of TCMP manifestations, including the most severe ones - AF and HF.

The incidence of heart rhythm disturbances in thyrotoxicosis varies in a wide range. Sinus tachycardia is the most common occurring in 42\%-73\% [Burggraaf J et al 2001, Cacciatori V, et al. 1996]. Tachycardia at rest and during sleep is a typical symptom in thyrotoxicosis. 
Heart rate at rest is known to be one of the most important reserve parameters and a predictor of fatal cardiovascular complications. Elevated resting heart rate is associated with higher mortality. Risk of death from all causes is three-times higher in subjects with resting heart rate of 90-99 versus 60 beats per minute. Predictive value of heart rate is higher in hypertensive people and those with coronary artery disease. High resting heart rate might cause worsening of hypertension, increases risk of acute cardiovascular events, coronary artery disease, fatal ventricular arrhythmias as the most common causes of a sudden death, blood pressure elevation and heart failure development. Sinus bradycardia is rather rare in thyrotoxicosis.

Atrial fibrillation takes second place among heart rhythm disturbances occurring in 9\%$23 \%$ of thyrotoxic patients compared to $0.4 \%-1.0 \%$ in general population (Petersen \& Hansen, 1988; Presti \& Hart, 1989; Parle et al., 1991; Frost et al., 2004). This is the most common cardiovascular complication of thyrotoxicosis), and is associated with high morbidity and mortality related to the thromboembolism (Staffurt et al., 1977). The complication rate varies widely and will be discussed later. Thus, Sandler and Wilson (1959) and Petersen and Hansen (1988) found $14 \%$ and $14.9 \%$ occurrence of atrial fibrillation, respectively, in the population of subjects without concomitant heart disease. In a study of women with different cardiac pathology atrial fibrillation was diagnosed in $67 \%$ of subjects with thyrotoxicosis (Ladenson, 1993).

Subclinical thyrotoxicosis is associated with the increased risk of atrial fibrillation (Auer et al., 2001). In the Framingham study at 20 -year follow-up the incidence of AF was $25 \%$ in patients with low TSH that is 3 times as high as in euthyroid patients (7\%) (Sawin et al., 1994). The prevalence of AF seems to be comparable in overt and subclinical thyrotoxicosis. According to Auer J. et al. (2001) it was $13.8 \%$ in patients with overt thyrotoxicosis, $12.7 \%$ in subclinical thyrotoxicosis, and $2.3 \%$ in euthyroid subjects (Auer et al., 2001). Extrasystole (5\%-7\%), paroxysmal tachycardia (0.2\%-3.3\%) and atrial flutter (approximately $1.4 \%$ ) rarely occur in thyrotoxicosis. 24-hour ECG monitoring studies demonstrated that atrial heart rhythm disturbances are typical for thyrotoxicosis while ventricular ones usually occur only in severe cases and in subjects with concomitant cardiovascular diseases (table1).

\begin{tabular}{|l|l|}
\hline Arrhythmias & Frequency (\%) \\
\hline Sinus tachycardia & $42-73$ \\
\hline Atrial fibrillation & $9-23$ \\
\hline Extrasystole & $5-7$ \\
\hline Atrial flutter & $1,2-2,3$ \\
\hline Paroxysmal tachycardia & $0,2-3,3$ \\
\hline AV-block & $2,7-5$ \\
\hline Sick sinus syndrome can develop in elderly people \\
\hline
\end{tabular}

Table 1. Arrhythmias in thyrotoxicosis

The evidence on left ventricular hypertrophy $(\mathrm{LVH})$ prevalence is not enough. On the one hand, some authors found the increase in left ventricular myocardium mass in both overt (Nixon et al., 1979; Auer et al., 2001; Dorr et al., 2005; Marcisz et al., 2006) and subclinical (Ching et al., 1996; Biondi, 1999, 2000; Auer, 2001) thyrotoxicosis. On the other hand, there are not enough studies considering $\mathrm{LVH}$ occurrence in thyrotoxicosis and factors contributing its development, geometry type (eccentric or concentric), and reversibility. 
According to the epidemiology study (1510 patients over 45 years of age) by M. Dörr et al. (2005) LVH was diagnosed in $57,1 \%$ of patients with thyrotoxicosis, compared to $10.5 \%$ in euthyroid individuals. Therefore, thyrotoxicosis was considered an independent predictor of LVH (odds ratio, 13.65; 95\% confidence interval, 2.83-65.75; $\mathrm{p}<0.01$ ). We have analyzed the occurrence of $\mathrm{LVH}$ and factors contributing its development and reversibility among inpatients with thyrotoxicosis. The LVH occurrence was $22.7 \%$ that is lower compared to the mentioned data. We included patients under 55 years of age without concomitant cardiovascular pathology, and this could be a possible reason for the lower prevalence (Babenko et al., 2011).

The increase of left ventricular myocardial mass index (LVMMI) was demonstrated in all studies regarding subclinical thyrotoxicosis (Ching et al., 1996; Auer, 2001), however, the values did not meet diagnostic criteria for left ventricular hypertrophy (Biondi, 1999, 2000).

Only women with overt thyrotoxicosis, unlike those with subclinical thyrotoxicosis, had high values of LVMMI (meeting LVH criteria) associated with the decrease of end-diastolic left ventricular volume and development of concentric LVH (CLVH), as reported by Donatelli M et al. (2003) on the other hand, the majority of studies demonstrate increase of both LVMMI and end-diastolic left ventricular volume and development of eccentric LVH (ELVH) (Levina, 1989; Marcisz et al., 2006; Babenko et al., 2007, 2008, 2011).

Concentric remodeling of left ventricle occurs in ST (Biondi et al., 2002; Donatelli et al., 2003), while eccentric remodeling is more common in overt thyrotoxicosis.

Prevalence of heart failure also widely varies - from $12 \%$ to $68 \%$ (Forfar et al., 1982; Hrnciar, 2002; Roffi et al., 2003); up to $90 \%$ of patients with thyrotoxicosis and HF develop AF (Siu et al., 2007).

Dilated TCMP as a threatening complication of thyrotoxicosis should be mentioned (Goland et al., 1999). Siu C-W. et al. (2007) reported 1\% occurrence of dilated thyrotoxic cardiomyopathy in patients with thyrotoxicosis, and one thirds of cases is irreversible. Thyrotoxicosis accounts for $1 \%$ of the reasons of dilated TCMP (Lutton et al., 2001).

Association between thyrotoxicosis and pulmonary hypertension ( $\mathrm{PH}$ ) has been described as early as in the beginning of 1980s (Mier et al., 1989) and the linking mechanisms are discussed till nowadays (Yanai-Landau et al., 1995; Barst \& Loyd, 1998; Menzel et al., 2000; Armigliato et al., 2006). Graves' (autoimmune) disease accounts for the most cases of PH: 47-65\% of patients with diffuse goiter have PH (Mercé et al., 2005, Armigliato et al., 2006; Yung et al., 2006), such a high prevalence can be explained by both severe form of thyrotoxicosis and autoimmune effects (Yanai-Landau et al., 1995; Chu et al., 2002). The occurrence of $\mathrm{PH}$ in subclinical thyrotoxicosis is almost unknown. According to our data, the prevalence of $\mathrm{PH}$ was $29 \%$ in ST, and $39.6 \%$ in overt thyrotoxicosis.

The prevalence of mitral valve prolapse is $18-41 \%$ in thyrotoxicosis compared to $6-20 \%$ in general population (Brauman et al., 1985, Kahaly, 1986, Evangelopoulou et al., 1999).

\section{The pathogenesis of thyrotoxic cardiomyopathy and its manifestations}

\subsection{Cardiovascular effects of thyroid hormones}

Thyroid hormones (TH) affect cardiovascular system through three main intracellular processes in cardiomyocytes: energy production (oxidative phosphorylation, glycolysis), protein synthesis and cardiomyofibril contractility (Dillmann, 1990; Klein et al., 2000, 2001). 
Alteration of energy production. Myocardial oxygen consumption increases in thyrotoxicosis. The number of mitochondria and their functional activity grows leading to the enhancement of adenosine triphosphate (ATP) production. However, in severe thyrotoxicosis, despite the increased oxygen consumption, the efficiency of its utilization decreases significantly. This follows altered oxidative phosphorylation resulted from the abnormal oxidative enzyme activity (decrease of myocardial ATP and creatine phosphate levels results in glycolysis activation) (Golber \& Kandror, 1972).

Direct and indirect effects on myocardial of abundant $\mathrm{TH}$ result in alteration of cardiomyofibril contractility. Direct effects involve transcription of some genes regulating function of cardiomyiocytes, vascular smooth muscle cells (SMC) and other structural units (Dillmann, 1990; Klein et al., 2000, 2001)(Fig.1). Triiodothyronine (T3) is a biologically active thyroid hormone that enters cardiomyocytes through specific transporters located in the cellular membrane, and binds thyroid hormone receptor (THR) in the nucleus (Dillmann,1990; Everts et al., 1996). Nowadays two types of THR are known: homodimer (THR/THR) and heterodimer - 9-cis retinoic acid receptor (THR/RXR). Active forms of these receptors affect gene expression through binding specific DNA response elements, so called thyroid hormonesensitive elements (TRE), located in the promoter region of T3-sensitive genes (Brent, 1994; Everts et al., 1996; Fazio et al., 2004). Gene transcription is augmented in the regions encoding alpha-myosine heavy chains (MNC-a), $\mathrm{Ca}^{2+}$-ATPase of sarcoplasmatic reticulum of the cardiomyocytes, $\mathrm{Ca}^{2+-} \mathrm{K}^{+}$-ATPase $\mathrm{Ca}^{2+-}$-uretic, beta1-adrenergic receptors, troponine $\mathrm{I}$ and $\mathrm{Ca}^{+}$-uretic peptide. Three types of myosine isoforms are distinguished: $\mathrm{V} 1$ consisting of the MNC-alpha/alpha chains, V2 - consisting of MNC-alpha/beta chains, and V3 - consisting of MNC-beta/beta chains. High levels of TH affect myosine isoform expression and lead to the enhanced synthesis of V1 isoform and decrease of V3 isoform. ATPase being more active in MNC-alpha/alpha myosine chains compared to MNC-beta/beta chains, the velocity of myofibril contractions increases (Klein \& Ojamaa, 2000, 2001) (Fig.1).

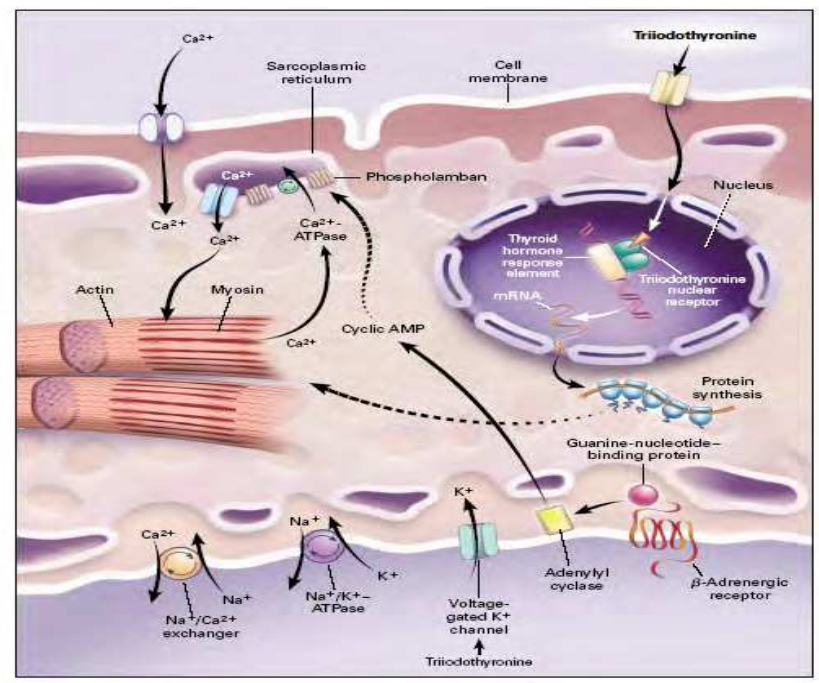

Fig. 1. Genom and nongenom effects of triiodthyronine in cardiomyocytes (from Klein\& Ojamaa, 2000) 
Indirect myocardial effect of abundant thyroid hormones include $\mathrm{Ca}^{2+}$ release from the intracellular depots and their subsequent reuptake by sarcoplasmic reticulum. The latter results from the enhanced $\mathrm{Ca}^{2+}$ ATPase (SERCA) expression and phospholamban suppression that are involved in the regulation of calcium intramyocyte concentration during cardiac cycle (systole and diastole). This leads to the increase of peak strain of cardiomyofibrils and shortening of the ventricular systole. Abundant thyroid hormone have been shown to induce expression of ryanodine receptors facilitating calcium $(\mathrm{Ca} 2+)$ release from sarcoplasmic reticulum, thus amplifying contraction and myocardial hypertrophy. Moreover, high levels of $\mathrm{TH}$ cause intracellular calcium mobilization directly affecting L-type of Ca2+-channels (Dillmann , 1990, Kiss et al., 1994; Klein \& Ojamaa, 2000, 2001)(Fig.1).

Therefore, high levels of $\mathrm{TH}$ contribute to the increased myocardial contractility through enhanced transcription of the genes encoding heavy chains of myocine beta-isoform and calcium-dependent ATPase, induced protein synthesis and augmented calcium and glucose myocardial consumption. In addition, thyroid hormones cause 50-70\% reduction of the systemic vascular resistance (SVR) through direct and indirect (increase of tissue thermogenesis and lactate hyperproduction) effects on vascular smooth muscle cells leading to the decrease of diastolic blood pressure (DBP) and the increase of end-diastolic left ventricular volume. In particular, $\mathrm{TH}$ are known to induce nitric oxide production causing vasodilatation through direct effects on vascular smooth muscle cells (Ishikawa et al., 1989; Klein \& Ojamaa, 2000, 2001; Vargas et al., 2006). Moreover, high levels of TH lead to the increase of circulating blood volume and venous return through the following mechanisms: aldosterone-renin system activation, enhancement of erythropoietin synthesis and renal sodium reabsorption (Jiménez et al., 1982; Marchant et al., 1993; Klein \& Ojamaa, 2000, 2001; Vargas et al., 2006). Renal juxteglomerular complex is activated when DBP is reduced (Büssemaker et al., 2003). In addition, T3 directly stimulates rennin substrate synthesis by liver (Ganong WF. 1982). The reduction of BP and SVR induces production of rennin and aldosterone resulting in the increase of circulating blood volume and end-diastolic volume that finally amplifies workload on heart (Klein \& Ojamaa, 2000, 2001; Vargas et al., 2006) (fig.2).

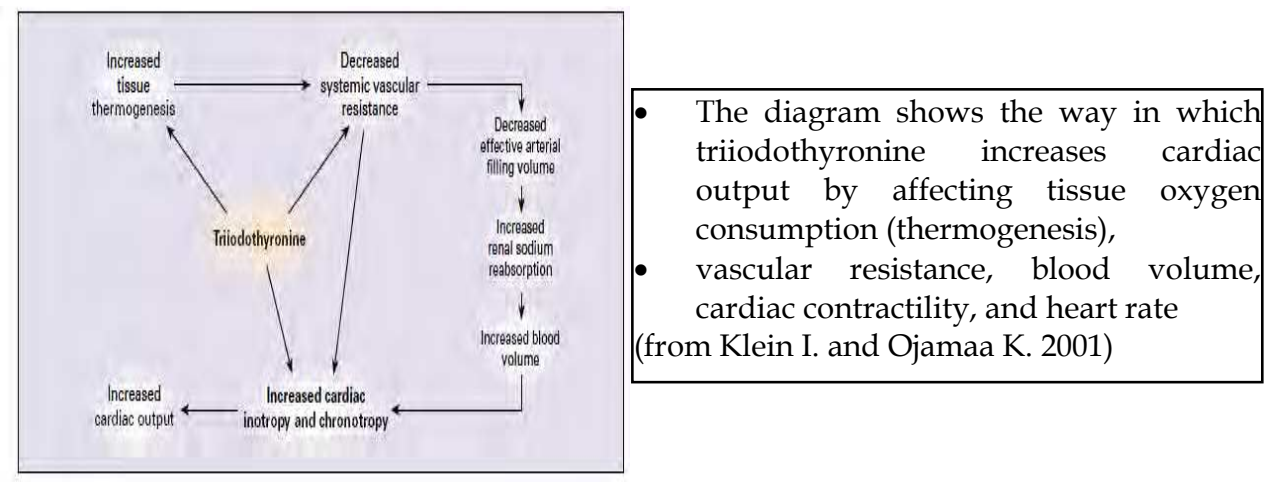

Fig. 2. Effects of Thyroid Hormone on Cardiovascular Hemodynamics 
Tachycardia is a typical feature of thyrotoxicosis (Nordyke, 1988; Cacciatori, 1996; Biondi, 2000). It is caused by the faster diastolic depolarization associated with the shortening of action potential of the sinoatrial node (Osman et al., 2002). In general, the TH effects on pacemaker and conductive cardiac system include acceleration of systolic and diastolic depolarization and shortening of action potential, as well as of refractory period of atrial and atrioventricular myocytes. The latter can result in the increase of AF occurrence in thyrotoxicosis. Other mechanisms include augmentation of the pressure in left atrium, ischemia and left atrial premature ectopic heart beats (Bielecka-Dabrowa et al., 2009).

Thyroid hormones regulate some myocardial genes encoding synthesis of ion channels $\left(\mathrm{K}^{+} / \mathrm{Na}^{+}\right.$-ATPase, $\mathrm{Ca}^{+} / \mathrm{Na}^{+}$- exchanger and potential-depending potassium channels) in cell membrane. These extranuclear effects involve the transport of aminoacids, glucose and calcium through cell membrane. Therefore, T3 can alter the activity of various ion channels (including sodium, potassium and calcium channels) located in cell membrane. (Bielecka-Dabrowa et al., 2009). In particular, phospholipid and Ca-dependent proteinkinase, cAMP-dependent proteinkinase, and Ca2+-calmodulin complex as the components of signal transmission system are involved in the T3 non-genomic cell effects. Thus, non-genomic actions of thyroid hormones include regulation of intracellular ion levels and their intracellular distribution. Changing transmembrane ion transport TH affect electrophysiologic features of cardiomyocytes (Kim \& Smith, 1984). T3-induced expression of L-type calcium channels leads to the following: less calcium ions enter cardiomyocytes through L-type channels, and action potential gets shorter (Freedberg et al., 1970).

The occurrence of atrial rhythm disturbances (atrial fibrillation, atrial premature heart beats) is higher at thyrotoxicosis, while the rate of ventricular arrhythmias is comparable with the one in general population (Osman et al., 2002). Atrial and ventricular myocardial sensitivity to thyroid hormones is different that can be the cause of the variation in atrial and ventricular arrhythmia prevalence. High atrial sensitivity to arrhythmogenic thyroid effects is due to the high $\beta$-adrenoreceptor density at the surface of atrial cardiomyocytes and to the different autonomic innervation of atria and ventricles (Polikar et al., 1993; Lombardi et al., 1994). Golf et al. (1985) found that $\beta$-adrenoreceptor density in right atria of human heart samplings is more than twice as high as in left ventricle. Higher $\beta$-adrenoreceptor density in thyrotoxicosis is associated with the greater catecholamine myocardial sensitivity. Androgens up-regulate expression of the $\beta$-adrenoreceptor genes leading to their density increase. This can explain higher susceptibility to heart rhythm disturbances in men compared to one of women.

Expression of potential-dependent $\mathrm{K}+$-channels in atria is $30 \%$ as high as in ventricles (Ojamaa et al., 1999). Expression of genes encoding both $\beta$-adrenoreceptor and potentialdependent $\mathrm{K}+$-channels depends on thyroid hormones that induce heart rhythm disturbances. Experimental mice studies demonstrated that duration of atrial action potential is shorter, and atrial delayed rectifier potassium current (ultra rapid - Ikur and slow - Iss) mediating repolarization is greater in thyrotoxicosis. RNA messenger level and expression of genes of K+-channels (Kv1.5 и Kv2.1) were shown to be higher in both atria that also leads to the greater density of delayed rectifier potassium channels (Ikur $и$ Iss) in hyperthyroidism. Thyroid effects on action potential and delayed rectifier potassium current are more expressed in right atrium compared to left one resulting in low interatrial difference of action potential duration (Bielecka-Dabrowa et al., 2009). 
Shortening of atrial action potential in thyrotoxicosis is associated with the shorter duration of effective refractory period facilitating re-entry foci developing. On the other hand, normal interatrial difference of action potential is of great importance for synchronous atrial contraction (regarding right-sided location of the sinus node). Spontaneous ectopic activity, particularly in left atrium, can increase leading to atrial heart rhythm disturbances (atrial fibrillation) when the interatrial action potential difference is reduced (Bielecka-Dabrowa, 2009).

Pulmonary veins are known to initiate paroxysmal atrial fibrillation that can be due to the increased spontaneous activity of that region. Shorter action potential of the cardiomyocytes located in pulmonary veins associated with the shortening of refractory period and facilitating re-entry onset was shown in experimental thyrotoxicosis models (Chen, 2002). Incubation with thyroid hormones increased spontaneous activity in pulmonary vein cardiomyocytes similar to its effect on sinoatrial node cells. Trigger activity is supposed to underlie spontaneous activity of pulmonary veins (Bielecka-Dabrowa et al., 2009).

\subsection{Pathogenesis of clinical manifestations of thyrotoxic cardiomyopathy}

Tachycardia in thyrotoxicosis develops due to the faster diastolic depolarization associated with the shortening of action potential of the sinoatrial node cells [Osman F et al. 2002]. Sinus bradycardia can develop in case of congenital disorders or sick sinus syndrome. The latter, as well as abnormal heart rhythm can result from the decreased reserves of the mediator acetylcholine.

Mechanisms of arrhythmogenesis are discussed in the former chapter. Pathogenesis of heart rhythm disorders is not clear, and the following pathogenetic factors are being discussed: toxic effects of thyroid hormones, increased sympathetic activity, reduced intracellular potassium level (Table 2).

\begin{tabular}{|l|l|}
\hline Effects of thyroid hormones & $\begin{array}{l}\text { Pathogenesis of influence of thyroid } \\
\text { hormones }\end{array}$ \\
\hline $\begin{array}{l}\text { Toxic influence on myocardium } \\
\text { (amplification cardiomyofibril contractility } \\
\text { and hypertrophy right atrium and left } \\
\text { ventricule of heart) }\end{array}$ & $\begin{array}{l}\text { Alteration oxidative phosphorylation. } \\
\text { Increase synthesis of protein, action of } \\
\text { enzymes, uptake of } \mathrm{O}_{2} \\
\text { Alteration power processes and changes of } \\
\mathrm{K}+/ \mathrm{Na} \text {-pump - increase velocity of } \\
\text { diastolic depolarization }\end{array}$ \\
\hline Increase sensitivity of SNS & $\begin{array}{l}\text { Increase of number of } \beta 1 \text {-adrenoreceptors } \\
\text { Decrease of intracellular pool of potassium } \\
\text { action of simpatoadrenal influence }\end{array}$ \\
\hline Change of vascular reactivity & Hyperrelaxations vascular myocites \\
\hline
\end{tabular}

Table 2. Pathogenesis of arrhythmias in thyrotoxicosis

Cardiac hyperfunction and increased adrenergic stimulation contribute the loss of intracellular potassium ions leading to heart rhythm disorders. Atrial dystrophic alterations associated with the structural myocardial heterogeneity are a confounding factor for atrial fibrillation. Exposure to any additional factor increasing myocardial heterogeneity can result in the complete myocardial uncoupling inducing heart rhythm disturbances. Atrial dilation, increased excitability of atria associated with the myocardial heterogeneity mediating 
functional conductive disorders underlie re-entry arrhythmias. Re-entry associated with the frequent focal activity are supposed to be the main mechanism of atrial fibrillation and flutter in thyrotoxicosis.

Increased cardiac output and reduced system vascular resistance (SVR) in thyrotoxicosis commonly lead to the amplification of pulse amplitude. Hemodynamics is altered in thyrotoxicosis as it has been mentioned above. The changes include elevation of heart rate, stroke and minute volumes, acceleration of blood flow, decrease of systemic vascular resistance (Fig.3), and resulting changes of blood pressure - systolic blood pressure is moderately increased, while diastolic blood pressure is within normal values or decreased. Thus pulse pressure goes up. The recent studies have demonstrated arterial stiffening and low SVR in thyrotoxicosis (Palmieri et al., 2004). Therefore, high levels of thyroid hormones cause systolic blood pressure (BP) elevation leading to isolated systolic hypertension in elderly patients with decreased arterial elasticity due to the atherosclerotic vascular lesion. The prevalence of isolated systolic hypertension is significantly higher in patients with thyrotoxicosis compared to euthyroid subjects (Marcisz et al., 2001). ST can resulting in an increase in nighttime systolic and mean blood pressure (Botella-Carretero et al, 2004). It appears to be one of the factors for LVH development in thyrotoxicosis.

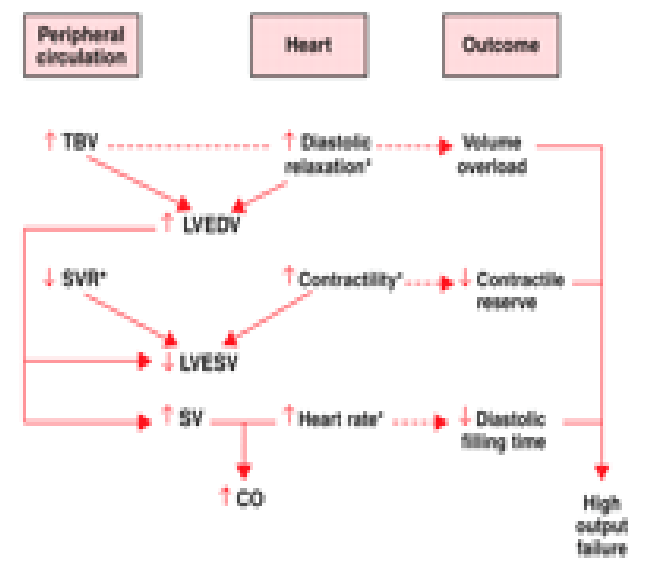

TBV- total blood volume;

LVEDV - left ventricular end diastolic volume; LVESV - left ventricular end systolic volume;

SV - stroke volume;

SVR - systemic vascular resistance;

$\mathrm{CO}$ - cardiac output;

$\uparrow$ increase; $\downarrow$ decrease.

Solid arrows indicate direct effects, and dashed arrows potential outcomes.

*Features for which T3 is directly responsible. (from A Toft, N Boon 2000)

Fig. 3. Effects of hyperthyroidism on the cardiovascular system and the possible outcomesEccentric type of LVH, as a result of low SVR and volume overload on the heart, is a typical feature of thyrotoxicosis. However, there are data showing both normal LV geometry (Levina, 1989; Babenko, 2008), and LVH (eccentric type (Levina, 1989; Marcisz et al., 2006; Babenko et al., 2008, 2011) as well as concentric one (Donatelli et al., 2003) in patients with thyrotoxicosis. It depends on the degree of cardiovascular damage influenced by the variety of factors that will be discussed later.

Reduced functional cardiac reserve and physical load tolerance are also common in thyrotoxicosis because tachycardia at rest prevents from the increase of heart rate and effusion fraction and from the SVR lowering at physical load.

Pulmonary hypertension (PH) (mean pulmonary pressure more than $25 \mathrm{mmHg}$ at rest and more than $30 \mathrm{mmHg}$ at load) is frequently reported at overt thyrotoxicosis. Pulmonary hypertension is often associated with valvular insufficiency (of tricuspid and mitral valves) 
due to the papillary muscles dysfunction (Whitner et al., 2005; Mercé et al., 2005). Patients with Graves' disease are more likely to have myxomatous degeneration and prolapse of mitral valve as a consequence of endocardium involvement compared to subjects with toxic multinodular goiter (Kahaly, 1987).

There are two possible mechanisms of valve involvement in Graves' disease. First mechanism includes induction of glycosaminoglycan synthesis in endocardium (similar to one in retrobulbar space in ophthalmopathy) leading to the valve cusp thickening (Kahaly, 1987). The second one implies the reduced tone of papillary muscles and their consequent overstretching resulting in prolapse of mitral valve cusps in left atrium during ventricular systole (Brauman et al., 1985).

Pulmonary hypertension develops due to Kitaev reflex - spasm of pulmonary arterioles leading to the increase of pulmonary resistance (Okura \& Takatsu, 1994). Thyroid hormones seem to affect pulmonary vessels in a rather different way compared to other vessels as they cause SVR reduction and circulating blood volume elevation. Blood pressure decreases in resistive vessels due to the vasodilatation effect of $\mathrm{TH}$, and increases in pulmonary vessels. This is possibly mediated by endothelial dysfunction (Okura \& Takatsu, 1994), and by the accelerated metabolism of pulmonary vasodilatation substances, such as acetylcholine, observed in patients with severe thyrotoxicosis (Okura \& Takatsu, 1994; Nakchbandi et al., 1999). In addition, autonomic imbalance, in particular, sympathetic hyperactivity and lower vagal tone (Burggraaf et al., 2001), caused by hyperthyroidism facilitates further pulmonary vasoconstriction and $\mathrm{PH}$ development. Autoimmune mechanism might be an additional factor for PH development (Yanai-Landau et al., 1995; Barst \& Loyd, 1998; James et al., 2002).

The diagnosis of heart failure in thyrotoxic patients is complex, because classic symptoms of heart failure including tachycardia and dyspnea, occur at the onset of hyperthyroidism due to both toxic effects of thyroid hormones on sinoatrial node and respiratory centre and intercostal muscles weakness. Heart failure (HF) develops in thyrotoxicosis because of the isotonic contraction (volume overload) of left ventricle and mixed overload (both volume and resistance overload) of right ventricle, thus right ventricular HF is more common for hyperthyroid state. Concomitant tricuspid valve insufficiency and regurgitation can worsen the situation (Whitner et al., 2005). Mitral valve prolapse is a common disorder in thyrotoxicosis, but it is rarely symptomatic.

Heart failure (HF) associated with thyrotoxicosis is more common in elderly rather than in young patients, and the mechanisms are better studied in aged group. Elderly subjects usually have co-existing cardiovascular diseases (coronary artery disease, valvular heart disease, arterial hypertension) leading to the functional and structural cardiac alterations, thus, myocardium contractility is easily impaired in case of increased myocardial load caused by hyperthyroidism. Elderly patients with heart rhythm disturbances, such as atrial fibrillation (AF), have the highest risk of HF development, because AF is usually associated with the increased heart rate followed by functional impairment of cardiomyocytes. Younger patients without cardiovascular pathology more often develop "high output heart failure" (Okura \& Takatsu, 1994). This type of HF is characterized by the myocardial hypercontractility. HF with low output and heart chambers dilation (dilated thyrotoxic cardiomyopathy) can develop in young patients in case of the long-lasting severe thyrotoxicosis (Manger et al., 1988; Soh \& Croxson, 2008). However, long duration of hyperthyroidism is not always evident, so the mechanisms are not fully understood. 
Autoimmune mechanisms were considered possible (Kahaly, 1987; Koshiyama et al., 1996), but the hypothesis was denied afterwards (Fatourechi \& Edwards, 2000). Two hypotheses are discussed nowadays. According to the first one two diseases are supposed to coexist in one individual - Graves' disease and idiopathic dilated cardiomyopathy (IDC). It is supported by their common pathogenesis - genetic predisposition determined by the HLA haplotypes (Limas \& Limas, 1989; Olson et al., 1992) and common association of IDC and autoimmune thyroid diseases (Marković et al., 2005). According to the second hypothesis long-lasting myocardial hypertrophy can transform into dilation, and heart chamber dilation can develop in untreated prolonged overt and even subclinical thyrotoxicosis (Soh \& Croxson, 2008).

Therefore, there are two main mechanisms of heart failure development in thyrotoxicosis: 1) tachycardia-induced HF with left ventricular dysfunction (left ventricular HF) is most common in patients with AF; 2) right ventricular HF resulted from volume overload of right ventricle due to the increased circulating blood volume and venous return is characterized by right ventricular dilation, enlargement of the tricuspid valve ring and tricuspid insufficiency and is frequently associated with pulmonary hypertension (Okura \& Takatsu, 1994; Xenopoulos et al., 1996; Whitner et al., 2005).

Three stages of thyrotoxic cardiomyopathy are defined: hyperkinetic (with preserved left ventricular function, but at physical load left ventricular effusion fraction does not increase), normokinetic (a compensatory stage with a reversible myocardial hypertrophy and preserved cardiac output), and hypokinetic stage (decompensation stage with low cardiac output and stroke volume, either reversible or irreversible heart chamber hypertrophy and dilation).

High occurrence of thromboembolic events (higher than in general population) is typical for thyrotoxicosis, and even in absence of AF. Endothelial dysfunction is a possible explanation for high rate of thromboembolism: altered vasodilation response to acetylcholine exposure (Faber et al., 2001, Napoli et al., 2001), as well as elevated level of humoral substances Von Willebrand factor (Cucuianu et al., 1987; Squizzato et al., 2007), plasminogen activator, endothelin (Baumgartner-Parzer et al., 1997) confirm thrombogenesis activation in thyrotoxicosis (Homoncik et al., 2007). ST has been recently shown to have a prothrombogenic effects (Horne et al., 2004; Erem, 2006). Increased activity of Von Willebrand factor (Coban et al., 2006) and elevated fibrinogen level (Dörr et al., 2001) were demonstrated at ST.

\section{Clinical manifestations and diagnostic tests of thyrotoxic cardiomyopathy}

The most common symptoms of thyrotoxic cardiomyopathy are palpitations, chest pain (cardialgia), dyspnea, irregular heart beats. Tachycardia and heart rhythm disturbances, systolic hypertension, orthostatic hypotension, heart enlargement, systolic murmur maximal over mitral valve and bubbling rales in case of HF are the most common findings at physical examination. Tachycardia at rest and during sleep and significant increase of heart rate at minimal physical exertion are typical for thyrotoxicosis. Tachycardia is position-independent and resistant to therapy by cardiac glycosides. Usually there is a direct relation between tachycardia intensity and the severity of diffuse goiter. Heart rate increases up to 120-140 beats per minute, and up to 160 beats per minute and more on movement, at physical and emotional exertion. Patients complain of pulsation in the neck, head and abdomen. Atrial fibrillation can develop in both clinical thyrotoxicosis and subclinical and latent forms. Thus, as a potential cause thyrotoxicosis should be excluded in all patients with persistent atrial fibrillation resistant to antiarrhythmic drugs. Thyroid dysfunction is the cause of $46 \%$ of arrhythmias of 
this type. Paroxysmal atrial fibrillation at the onset of thyrotoxicosis usually turns into permanent form with the progression of the disease. There is a particular form of thyrotoxicosis characterized by paroxysmal atrial fibrillation with normal sinus rhythm or bradycardia without any other symptoms of thyrotoxicosis. The level of thyroid hormones is crucial to make the diagnosis in such cases. Physical examination reveals tachycardia and ascending apex beat. The cardiac borders are expanded to the left, the heart sounds are intensive, first heart sound is loud, and systolic murmur can be heard. Systolic blood pressure is increased, while diastolic blood pressure is within normal values or decreased (resulting in high pulse pressure, or pulse amplitude). Water retention is usual, and edema can be found even in patients with preserved ejection fraction. If the symptoms are persistent after successful treatment of thyrotoxicosis coexistent cardiovascular pathology should be excluded. In otherwise healthy individuals palpitations are associated with female sex and age older than 50 years, dyspnea is linked to female sex and increased level of T4>30 pmol/l. After euthyroid state restoration dyspnea can persist in smoking subjects (Osman et al., 2007).

Heart failure is associated with the pleural effusions, and right ventricular heart failure is more prevalent in diffuse goiter (Levina, 1989). Patients with dilated thyroid cardiomyopathy reveals cardiomegaly and pulmonary congestion (Soh \& Croxson, 2008) and all spectre clinical sins of heart failure.

Diagnosis. ECG changes include high, sharp $\mathrm{P}$ and $\mathrm{T}$ waves, atrial fibrillation, and extrasystole. ST segment depression and $\mathrm{T}$ wave inversion might be also present without angina pectoris, and are due to metabolic changes. ECG changes are reversible if the thyroid hormone levels are normalized.

Dilation of both ventricles and conus pulmonalis expansion are typical radiological signs in severe thyrotoxicosis. Conus pulmonalis expansion and right ventricle enlargement form mitral configuration of the heart. However, there is no left atrium enlargement at oblique view unlike the changes in mitral stenosis.

Pulmonary edema is another radiological sign in thyrotoxicosis. Echocardiography finds left ventricular hypertrophy including posterior wall and interventricular septum thickening, increase of end-diastolic volume and of left ventricular myocardial mass. Mitral valve prolapse is a common finding in thyrotoxicosis. The dilation of all heart chambers and decreased systolic function of left ventricle can be found in dilated thyrotoxic cardiomyopathy.

Perfusion scintigraphy of the heart with thallium chloride 201 demonstrates diffuse or small-focal decrease of cardiomyocyte metabolic activity. This diagnostic method enables assessment of the intact cardiomyocytes, and is helpful to exclude myocardial damage at early stages of the disease.

Stress-echocardiography is useful for assessment of functional myocardial reserve.

Increased levels of thyroid hormones and TSH suppression are crucial parameters to confirm the underlying cause (thyrotoxicosis) of the clinical findings.

\section{The impact of various factors in clinical manifestations of thyrotoxic cardiomyopathy}

\subsection{Left atrial dilation and atrial fibrillation}

Right atrial hypertrophy associated with dilation and verified by ECG was found in 30.5\% of patients with thyrotoxicosis, left atrial hypertrophy - in $13.7 \%$, and the hypertrophy of both atria was found in $11.8 \%$ patients. 
Atrial fibrillation occurrence in thyrotoxicosis widely varies depending on the studied population. Age, sex, severity and intensity of hyperthyroidism, and concomitant cardiovascular diseases affect the incidence of atrial fibrillation.

Age-dependence is well-known: the risk of AF development increases as patients get older. Thus, at 10 -year follow-up the incidence of atrial fibrillation was $25 \%$ in patients over 65 years of age with thyrotoxicosis, even its subclinical form, and this is threefold risk of patients with normal TSH (7\%). The prevalence of AF was $21 \%$ in total cohort patients with clinical thyrotoxicosis (Iwasaki T et al, 1989), and 31\% in subjects over 40 years old, while there was no single case of AF in individuals younger than 40 years. In another study $25 \%$ of thyrotoxic subjects over 60 years old had AF compared to 5\% in younger group (Agner et al., 1984). Regression analysis performed by Nakazawa H. (2000) demonstrated a clear age-dependence of AF prevalence increasing stepwise till the maximal values in patients older than 70 years.

Male sex is another risk factor for AF (Staffurt et al., 1977). Toft A. \& Boon N (2000) reported up to $50 \%$ prevalence of $\mathrm{AF}$ in males aged 60 years with hyperthyroidism.

The impact of the duration of thyrotoxicosis is less investigated. AF is quite rare (less than $2 \%$ ) at onset of overt thyrotoxicosis (Nakazawa et al., 2000) and in patients under 40 years old without concomitant cardiovascular diseases Toft A. \& Boon N (2000). The following factors are considered the most significant for AF development in young subjects without concomitant cardiovascular pathology: thyrotoxicosis duration $(\mathrm{p}=0,0005, \mathrm{OR}=72(6,6-794))$, male sex $(\mathrm{OR}=10,4(3,0-36,0), \mathrm{p}=0,0003)$, and heart rate $(\mathrm{OR}=1,07(1,03-1,11) \mathrm{p}=0,0001)$ Babenko et al., 2011). These factors are also important in case of coexistent heart pathology. Having examined 40628 patients with thyrotoxicosis Frost (2004), showed that the highest risk of AF is associated with male sex, coronary artery disease and congestive HF. J. Mercé et al. (2005) found higher occurrence of AF in thyrotoxic patients with symptomatic insufficiency of either mitral or tricuspid valves $(40 \%$ vs. $14 \%, P=0,02$ in mitral regurgitation, and $86 \%$ vs. $3 \%, \mathrm{P}=0,01$ in tricuspid regurgitation).

Data on the effect of thyrotoxicosis intensity (overt or subclinical) are controversial. Auer et al.(2001) reported similar relative risk of AF in both subclinical and overt thyrotoxicosis (12,7 \% and 13,8\%, respectively). According to other data, AF occurrence in ST was $7 \%$ compared to $16 \%$ in clinical thyrotoxicosis (Babenko et al., 2011). There is an association between atrial fibrillation occurrence and type of predominantly elevated thyroid hormone (T3 or T4). Thus, atrial fibrillation occurs in 36\% patients if $\mathrm{T} 3$ is elevated, in $13 \%$ - if $\mathrm{T} 4$ is increased, and in $21 \%$ - if the levels of both thyroid hormones are high. Therefore, the highest occurrence of atrial fibrillation was observed in the thyrotoxicosis due to isolated T3 excess. However, the difference could be explained by the different duration of the disease: isolated T3 thyrotoxicosis develops in nodal thyroid autonomy that is characterized by the long-term asymptomatic period and slow increase of thyroid hormone level at first leading to subclinical thyrotoxicosis, and then to T3 thyrotoxicosis. Conversion to sinus rhythm was reported only in $19 \%$ of patients with atrial fibrillation and at subclinical thyrotoxicosis. Risk factors for atrial fibrillation are listed in table 3.

Standard ECG could be used to identify high-risk patients at subclinical thyrotoxicosis: those with a short P-R interval who are predisposed to reentrant atrioventricular nodal tachycardia (Biondi B et al, 1998) and those with higher maximum $\mathrm{P}$ wave duration and $\mathrm{P}$ wave dispersion who are predisposed to AF (Aras D et al, 2005). In predisposed patients (those with two functionally distinct AV nodal conduction patterns), 1-thyroxine (1-T4) suppressive therapy may increase the occurrence of reentrant-atrioventricular nodal 
tachycardia because of the enhanced atrial excitability, which increases the number of atrial premature beats, and the shortening of the refractory period of the conducting tissue (Biondi $\mathrm{B}$ et al, 1998). Moreover, by measuring $\mathrm{P}$ maximum and $\mathrm{P}$ wave dispersion values, one could theoretically identify the patients with ST that are at high risk of AF (Aras D et al, 2005). Holter ECG could be performed before starting 1-T4 treatment in doses that suppress TSH to potentially identify patients who may be less tolerant to 1-T4 treatment.

\begin{tabular}{|l|}
\hline Age \\
\hline Male sex \\
\hline Duration of hyperthyroidism \\
\hline Heart rate \\
\hline Concomitant cardiovascular diseases \\
\hline
\end{tabular}

Table 3. Risk factors for atrial fibrillation development.

\subsection{Left ventricular hypertrophy}

LVMMI is influenced by T3 level, systolic BP level and body mass index in patients with thyrotoxicosis (Marcisz et al., 2006). Eccentric left ventricular remodeling is predominant, and treatment and restoration of euthyroid state is not associated with LVMI normalization (9-month follow-up).

Sex also affects the occurrence of left ventricular hypertrophy (LVH) in thyrotoxic patients: the incidence of $\mathrm{LVH}$ is higher in men than in women $(21,3 \%$ vs. $30,6 \%$, respectively, $\mathrm{OR}=3,6 ; \mathrm{CI}(1,8 ; 7,4) ; \mathrm{p}=0,05)$. Eccentric type of $\mathrm{LVH}$ was present in $18,2 \%$, and concentric one - in 4,3\%, concentric remodeling occurred in 7,4\% of patients (Babenko et al., 2011).

$\mathrm{LVH}$ rate increases in older population, however, the age-dependence is different in men and women. Correlation between LVMI and age is significant in general population $(\mathrm{r}=0,22$, $\mathrm{p}=0,0007)$, and in females $(\mathrm{r}=0,27, \mathrm{p}=0,0002)$, compared to non-significant association in males $(r=0,10, p=0,58)$. More precise analysis showed that these parameters are related nonlinearly, and variability of LVMI increases in older subjects. Non-linear regression analysis defined an age threshold of 44 years: LVMI does not change up to this limit, and increases rapidly in older subjects for more than $10 \mathrm{~g} / \mathrm{m}^{2}$. It is even more evident in females: mean LVMI is $90 \pm 20 \mathrm{~g} / \mathrm{m}^{2}$ in women younger 44 years, and $105 \pm 25 \mathrm{~g} / \mathrm{m}^{2}$ - in older ones. The prevalence of left ventricular hypertrophy increases significantly from 11 up to $36 \%$ $(\mathrm{OR}=4,4$; CI $(2,1 ; 9,1), \mathrm{p}=0,0002)$. Similar LVMI increase is observed in males - from $117 \pm 27$ to $124 \pm 36 \mathrm{~g} / \mathrm{m}^{2}$, but changes of LVMI values and $\mathrm{LVH}$ prevalence are not significant because of the high LVMI variability (and smaller group) $(p=0,63)$ (Babenko et al., 2011).

Atrial fibrillation is another factor associated with both LVH presence and severity (LVMI value) (Babenko et al., 2011).

The factors affecting left ventricular hypertrophy in subclinical thyrotoxicosis are not completely established. Shapiro L.C. et al. (1997) did not find LVH development in longlasting ST $(9,2 \pm 5,4$ years) and very low TSH $-0,001 \mathrm{mIU} / 1$ in subjects with endogenous ST (mean age $45 \pm 10$ years (27-63)). Biondi B. et al. (2000) reported significant increase of LVMI $\left(162 \pm 24 \mathrm{~g} / \mathrm{m}^{2}\right)$ in an age-matching group (43 \pm 9 years (27-69)) with endogenous ST but with higher TSH values $(0,15 \mathrm{mIU} / \mathrm{l})$ and shorter duration of subclinical thyrotoxicosis (6 months), however, it should be mentioned that $80 \%$ of the group were women. The same authors (Biondi et al., 1999) also demonstrated positive correlation between LVMI and duration and dozing of L-thyroxin treatment in patients with endogenous ST. There is 
evidence of different underlying mechanisms of LVH development in clinical and subclinical thyrotoxicosis (Donatelli et al., 2003), although the degree of LVH is comparable. LVH was shown to develop even in ST with normal heart rate, blood pressure and systolic left ventricular function that indicates the direct trophic effect of $\mathrm{TH}$.

LVMI is higher in patients with subclinical thyrotoxicosis of variable etiology (iatrogenic, autoimmune hyperthyroidism caused by subclinical Graves' disease and nonimmune hyperthyroidism at pretoxic thyroid adenoma) compared to age- and sex-matching control group ( $p<0,005$ ). LVH prevalence is $39 \%$ (43 patients, $45,5 \%$ in males and $38,4 \%$ in females) in ST. There was no difference in etiology and duration of ST. Male sex is a risk factor for LVH in ST as well as in clinical hyperthyroidism (Babenko et al., 2011).

Age and systolic blood pressure are two other risk factors for left ventricular hypertrophy (Babenko et al., 2007). Therefore, risk factors for left ventricular hypertrophy and atrial fibrillation development are different (Table 4)

\begin{tabular}{|l|}
\hline Age \\
\hline Male sex \\
\hline Duration of thyrotoxicosis \\
\hline Systolic blood pressure \\
\hline Concomitant cardiovascular diseases \\
\hline
\end{tabular}

Table 4. Risk factors for left ventricular hypertrophy

\subsection{Pulmonary hypertension}

J. Mercé et al. (2005) analyzed data on pulmonary hypertension and its risk factors. Pulmonary hypertension (PH) occurs in $41 \%$ of thyrotoxic patients. Patients with thyrotoxicosis and symptomatic mitral or tricuspid insufficiency have higher pulmonary pressure: 53,8 vs. $34,9 \mathrm{mmHg}, \mathrm{P}=0,0001$ in mitral insufficiency, 43,9 vs. $35,12 \mathrm{mmHg}, \mathrm{P}$ $=0,07$ in tricuspid insufficiency. There is no relationship between $\mathrm{PH}$ and following factors: age, sex, the cause of hyperthyroidism, cardiovascular or other symptoms, heart rate and type of heart rhythm disturbance, the level of thyroperoxidase antibodies, TSH, and TH. Pulmonary hypertension persisted in $16 \%$ of subjects after euthyroid state restoration. We found $39 \%$ prevalence of $\mathrm{PH}$ in thyrotoxicosis, and pulmonary pressure lowered till normal values in $61.5 \%$ of patients after persistent euthyroidism was achieved. The increase of pulmonary pressure was associated with free thyroxine level before treatment $(\mathrm{r}=0,5, \mathrm{p}=0,0001)$, age $(\mathrm{r}=0,25, \mathrm{p}=0,002)$ and endothelial vasodilatation function (the lower the response in reactive hyperemia test, the higher pulmonary pressure, $r=-0,37, p=0,002)$. Right ventricular diameter positively correlated with pulmonary pressure $(\mathrm{r}=0,36, \mathrm{p}=0,0001)$, and was considered a predictor of $\mathrm{PH}$ as there was $100 \%$ elevation of pulmonary pressure in patients with initial right ventricular dilation and normal pulmonary pressure.

According to our data pulmonary hypertension occurs in $29.6 \%$ of patients with subclinical thyrotoxicosis. The occurrence was highest $(37,5 \%)$ in subjects with endogenous nonimmune ST (autonomic thyroid nodules). Free T3 and the degree of TSH suppression had the greatest impact. Thus, there was a positive correlation between pulmonary pressure and free T3 level $(\mathrm{r}=0,56, \mathrm{p}<0,001)$, and negative - with TSH $(\mathrm{r}=-0,34, \mathrm{p}<0,005)$. Higher level of T3 appears to be the reason of the greater elevation of pulmonary pressure in nonimmune endogenous ST (Babenko et al., 2008). 


\subsection{Heart failure}

Risk factors of heart failure (HF) development in thyrotoxicosis are not well understood. As reported by Siu C-W et al. (2007), $5.8 \%$ thyrotoxic patients developed heart failure, and AF was an independent predictor of HF (OR 37,4, (CI 9,72- 144,0, p <0,001)). 47\% had left ventricular dysfunction ( $\mathrm{EF}<50 \%$ ) that was more severe in patients with the lowest serum TSH level. Male sex was related with heart failure development (OR 26,6 (CI 2,6-272,5), p = $0,006)$. Left ventricular dysfunction and heart chamber dilation were present in one third of patients with restored euthyroidism. Patients with preserved output (ejection fraction 55\% vs. $30 \%, \mathrm{p}<0.001$ ) and lower functional class of heart failure (NYHA) demonstrate better outcome (reverse changes) (1.2 (0.1) vs. 2.5 (0.2), p <0.001).

The following main predictors of HF development in thyrotoxicosis (Babenko et al., 2011) are defined by multifactorial analysis: systolic blood pressure and free T3 level before treatment, dilation of left ventricle and to a lesser extent of other heart chambers.

To sum up, duration of hyperthyroidism, sex (male) and age are the main predictors of development of thyrotoxic cardiomyopathy and its clinical manifestations (left atrial dilation, atrial fibrillation, $\mathrm{LVH}, \mathrm{HF}$ ).

\section{Predictive and therapeutic approach to thyrotoxic cardiomyopathy based on its risk stratification}

Therapeutic approach should consider the factors affecting developing of thyrotoxic cardiomyopathy (TCMP) because persistent cardiac alterations have negative impact on cardiovascular prognosis.

European guidelines on management of toxic diffuse goiter offer the following strategy: conservative treatment by antithyroid drugs should be administered for 18 months with the following withdrawal in case of remission achieved, otherwise non-medication (irreversible) treatment is strongly recommended.

Persistent euthyroid state achievement is of great importance in management of thyrotoxic cardiomyopathy symptoms, while greater duration and older age are associated with worse cardiovascular prognosis. In our study (Babenko et al., 2011) median duration of hyperthyroidism was $10(6 ; 16)$ months in women without left ventricular hypertrophy $(\mathrm{LVH})$ and $10(6 ; 15)$ in men, while it was up to $20(9 ; 40)$ months in women with LVH $(p=0,006)$ and up to $19(12 ; 30)$ in men with left ventricular hypertrophy $(\mathrm{p}=0,09)$. Therefore, left ventricular hypertrophy risk is low in thyrotoxicosis lasting for less than 10 months, but it is significantly higher in more prolonged disease. In addition, left ventricular hypertrophy risk was higher in women over 44 years old, but it was age-dependent in men. Age and sex differences should be considered in clinical practice, and early non-medication treatment (after 8-10 months of antithyroid therapy) should be recommended in all male subjects and in women older 44 years. On the other hand, left ventricular hypertrophy in a newly diagnosed thyrotoxicosis is the sign of the long-lasting pathology, and its total duration is longer than mentioned periods. These subjects should undergo irreversible treatment earlier. Systolic hypertension worsens cardiovascular prognosis and it must be effective curation.

The choice of non-medication therapy- surgery or radioiodine intervention - is not easy in patients with thyrotoxic cardiomyopathy. Surgical treatment is associated with the high peri- and intraoperative risk, and radioiodine therapy is linked to the worse prognosis. The latter is due to the radioactive thyreoiditis development leading to the "leakage" 
thyrotoxicosis, causing atrial fibrillation recurrence, vasospasm and cardiovascular events (myocardial infarction, stroke, pulmonary thromboembolism). I131 treatment is associated with the high early mortality at 1-2 months after intervention that coincides with the time of radioactive thyreoiditis onset.

Because increasing cardiovascular risk is present in older ST patients, treatment of endogenous ST (antithyroid drugs or radioiodine) is recommended to these patients. Restoration of euthyreoidism improves cardiovascular parameters (reduction in heart rate and the number of atrial and ventricular premature beats, LVMI and cardiac output) (Biondi and Cooper, 2008). At older patients it is necessary to avoid whenever possible exogenous ST. Recent guidelines do not recommend suppressive therapy with l-T4 (to suppress serum TSH to below 0.1 $\mathrm{mIU} /$ liter) of benign thyroid nodules. Long-term suppressive therapy with serum TSH concentrations below $0.1 \mathrm{mIU} /$ liter is recommended for DTC patients with persistent disease and a high risk of recurrence. Beta-blockade might be considered in high-risk thyroid cancer patients with adrenergic hyperresponsiveness to 1-T4 (Biondi et al, 2005).

Symptomatic therapy of thyrotoxic cardiomyopathy should consider underlying pathogenetic mechanisms. Regarding high $\beta$-adrenoreceptor density, tachycardia due to increased adrenergic activity and volume heart load, $\beta$-blockers and diuretics should be administered. Diuretics are of great importance in patients with congestion symptoms (edema, dyspnea). Beta-blockers do not influence the release of thyroid hormones and are used to decrease sympathetic hyperactivity, thus leading to the reduction of cardiac load and myocardial oxygen demand. The choice of $\beta$-blocker is a highly debated question till nowadays. On the one hand, antithyroid activity of non-selective $\beta$-blockers (e.g. propranolol) is well-known . There is evidence that beta-blockers (propranolol) affect metabolism of thyroid hormones by inhibition of deiodinase type 2 in different tissues including cardiomyocytes and contributing transformation of T4 to inactive T3 - reversible hormone (Fig 4).
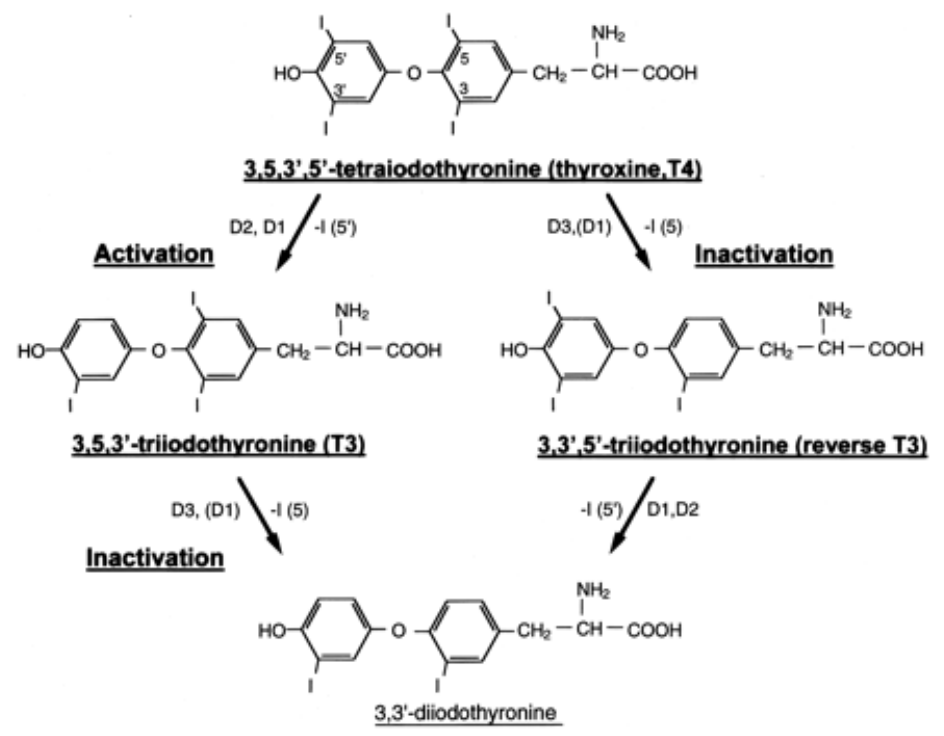

Fig. 4. Structures and interrelationships between the principal iodothyronines activated or inactivated by the selenodeiodinases (from A. Bianco, 2002) 
Deiodinases are the selenoenzymes regulating thyroxine (T4) transformation into triiodothyronine (T3) (Bianco et al. 2002, Kohrle, 1999). Type 1 deiodinase (D1) is present in liver, kidneys and thyroid gland (Bianco et al. 2002, Peeters et al. 2003) and is supposed to play the key role in active circulating hormone T3 production (Kohrle, 1999). Type 2 deiodinase (D2) enables T3 production in the following tissues: central nervous system, pituitary gland, brown adipose tissue, myocardium, somatic muscles, osteocytes; and it is less expressed in liver and kidneys (Bianco et al. 2002, Kohrle, 1999, Peeters et al 2006). Thus, D2 plays the key role in local tissue T3 production, however D2 activity in somatic muscles makes a major contribution to serological markers output (Peeters et al, 2006, Maia, 2005) . Therefore, free T3 concentration in tissues depends on both T4 synthesis in thyroid gland and, correspondingly, circulating T4 concentration, and deiodinase activity in tissues. D1 contribution varies from $15-39 \%$ in the most of studies to $80 \%$ (Bianco et al. 2002, Pilo, 1990) (fig.5). T3 tissue concentration less depends on plasma thyroid hormone levels as D2 activity can contribute to a larger T3 tissue output. As a result, T3 concentration differs a lot in various organs and tissues. For example, T3 saturation usually is about $50 \%$ in liver and kidneys while it amounts to $95 \%$ in central nervous system (Bianco A et al. 2002). According to scientific data, type 2 deiodinase activity rises many times in some tissues in patients with Graves' disease. Thus, high D2 activity was revealed in thyroid tissue extracted from patient with Graves' disease at surgery (Salvatore et al, 1996). There are data showing that thyrotoxicosis clinical manifestations do not always correlate with circulating thyroid hormone concentration. B.Biondi et al. (2000) using questionnaire (with special symptoms scale) revealed manifested thyrotoxic symptoms in some patients with subclinical thyrotoxicosis. Cardiovascular examination of these patients (Echocardiography (EchoCG), bicycle ergometry, electrocardiography, etc.) also revealed severe pathologic changes (high atrial arrhythmia prevalence, left ventricular hypertrophy, diastolic dysfunction).

Heart lesions similar to ones described in thyrotoxicosis patients were revealed in experiments involving mice with induced high D2 gene expression activity in cardiomyocytes (Pachucki et al, 2001).

Based on these data it was supposed that the severity of many pathologic changes including cardiovascular abnormalities in patients with Graves' disease can be associated with both circulating hormone level and deiodinase-dependent T3 tissue production. In particularly, energy consumption in skeletal muscles was shown to correlate with free thyroxin (FT4) and TSH circulating levels, but not with free triiodothyronine level (FT3) (Canani et al, 2005). Type 2 deiodinase is assumed to play the key role in T3 tissue concentration changes, at least, in the tissues (such as myocardium, vessel wall) with high D2 gene expression.

Latter studies showed clinical impact of certain polymorphisms of deiodinase genes. One of these is found in humans and includes D2 polymorphisms characterized by threonine (Thr) changed for alanine (Ala) in codone 92 (D2 Thr92Ala).

Ala/Ala homozygous patients demonstrate lower D2 tissue activity compared to Ala/Thr heterozygous and Thr/Thr homozygous patients. Therefore, this polymorphism can decrease T3 effects in tissues with high D2 gene expression (Bianco et al. 2002, Kohrle, 1999). Therefore, this polymorphism is suggested to influence clinical manifestations and the severity of heart damage in patients with thyrotoxicosis.

Our study shows that Thr92Ala D2 polymorphism can impact clinical course of Graves' disease and heart damage (Grineva et al, 2009). 


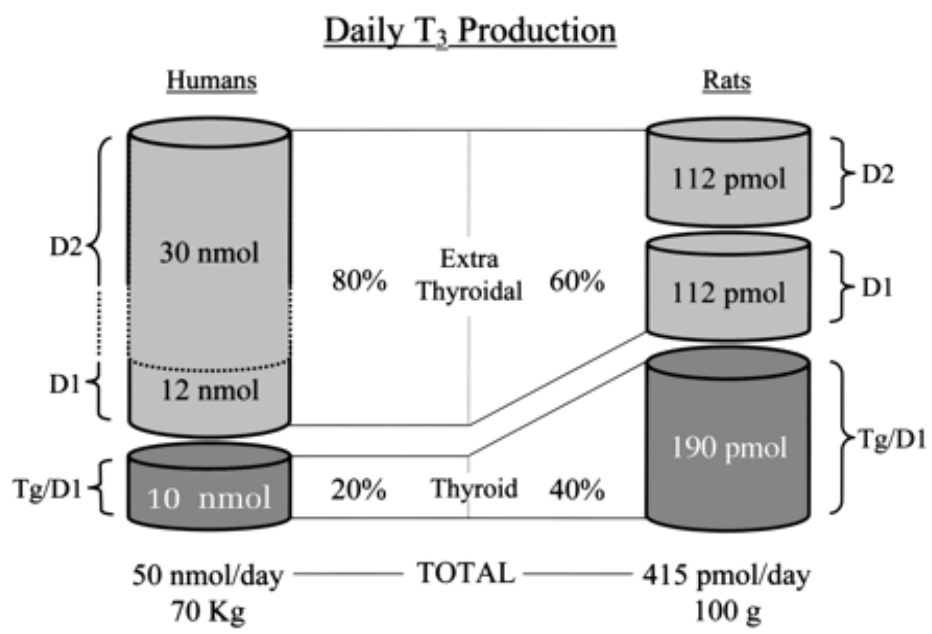

Fig. 5. Pathways of $\mathrm{T}_{3}$ production in humans and rats. The dotted lines in the cylinder representing human extrathyroidal production reflect the uncertainty about the exact contributions of D1 and D2 to this pool. Values given are based on the studies cited in the text. Values for rats are normalized to $100 \mathrm{~g}$ body weight (from A. Bianco, 2002)

T3 level and T3/T4 index before treatment are considered as predictors of Graves' disease remission. We show significant differences between these parameters depending on Thr92Ala D2 polymorphism occurrence prove its role as a predictor of Graves' disease prognosis. It is confirmed by considerable difference in disease duration in groups of patients with various genotype. Thr92Ala D2 polymorphism seems to be protective as disease duration and relapse prevalence were considerably lower in Ala/Ala homozygous patients. This leads to inhibition of T3 effects in tissues with high D2 expression, such as: heart, thyroid and pituitary gland. Our data prove that Thr92Ala D2 polymorphism can impact clinical manifestations of Graves' disease including thyroid volume gain and heart rate. Ala/Ala homozygous patients reveal lower heart rate than heterozygous and Thr/Thr homozygous ones. All these clinical features (lower hormone level and heart rate, less thyrotoxicosis duration) contribute to less severe thyrotoxic cardiomyopathy in Ala/Ala homozygous patients.

Ala/Ala genotype of D2 polymorphism is also protective lowering total LVH risk and negative prognostic types of remodeling (concentric hypertrophy and dilatation of chambers), in particular. Therefore, medications leading to the decrease of deiodinase 2 activity could result in the reduced clinical manifestations of TCMP, however, no evidence is still available (Grineva et al, 2009).

However, only high doses of propranolol (more than $160 \mathrm{mg}$ daily) can significantly decrease T3 plasma level (for approximately 30\%), and it can result in severe reduction of blood pressure in the majority of patients (normotensive ones) as far as only some of them develop systolic hypertension.

On the other hand, $\beta 1$-adrenoreceptor density is the first to be increased in thyrotoxicosis. Selective $\beta 1$-blockers are supposed to be more effective. However, the advantage of any certain $\beta$-blocker in TCMP management is not evident, thus, the choice is based on the other advantages, e.g. dosage: once- or twice-daily drugs are preferred. It should be stated that 
elderly subjects as well as patients with low output HF and low blood pressure level are at high risk of hypotension (in particular, postural hypotension) development when treated by beta-blockers. (15)Low doses of cardioselective beta-blockers - betaxolol, atenolol, metoprolol - are preferable in this group of patients and in subjects who have relative contraindications (eg. bronchial obstruction) to beta-blockers. Beta-blockers are usually administered while euthyroid state is achieved, and then the doses are gradually diminished and withdrawn, in some cases if necessary low doses are maintained for a long time. The latter includes sustained tachycardia resistant to antithyroid therapy, heart rhythm disturbances such as extrasystole and atrial fibrillation; heart failure due to the forceful cardiac contractions. Beta-blockers are also used before the effects of antithyroid drugs or radioiodine are apparent, as well as in thyrotoxic storm and as a part of preoperative care.

Combination therapy by beta-blockers and antithyroid drugs has certain cardiovascular benefits compared to monotherapy. However, individual beta-blocker susceptibility should be assessed. Decrease of heart rate and absence of side effects are the main signs of the correct dosing. Significant positive clinical effect is usually apparent after 5-7 days of therapy by beta-blocker, including reduction of sympathetic hyperactivity and related symptoms, decrease of heart rate (in sinus rhythm as well as in atrial fibrillation), extrasystoles are less frequent or disappear, in some cases atrial fibrillation converts to sinus rhythm.

In patients with exogenous ST, the administration of a beta-blocking drug reduced the increased heart rate and left ventricular mass (Fazio et al, 1995). Therefore, beta-adrenergic blocking drugs should reduce the cardiovascular risk, and thus they might be considered for patients requiring long-term TSH suppressive therapy, especially symptomatic patients with high-risk thyroid cancer in which more aggressive TSH suppression may be required (Biondi and Cooper, 2008).

Cardiac glycosides usually are not indicated in thyrotoxicosis even in patients with severe HF. They lead to systole enhancement, diastole prolongation, being vagotropic they slow down cardiac conductivity, in particular atrioventricular conduction. Thyrotoxicosis is characterized by hyperkinetic hemodynamics, delayed atrioventricular conduction, and cardiac glycosides are even dangerous as digitalis intoxication easily develops. Thyrotoxic patients are usually resistant to antiarrhythmic drugs, and amiodarone (containing one third of iodine) can induce severe therapy-resistant thyrotoxicosis, including thyrotoxic crisis.

Efficacy of AF treatment depends on several factors. Younger age (under 50 years), absence of HF symptoms or other coexistent cardiovascular pathology, short duration ( $<4$ month) and paroxysmal AF, normal systolic blood pressure and fast hypothyroidism achievement by antithyroid medication are the main predictors of sinus rhythm restoration that is observed in about one third of patients. Spontaneous conversion to sinus rhythm usually occurs at 3-month persistent euthyroid state. Otherwise standard guideline-based management of atrial fibrillation should be applied. The indirect relation between probability of successful cardioversion and duration of atrial fibrillation from any cause should be considered before the procedure (either pharmacologic or electrical cardioversion). Warfarin should be prescribed in patients with persistent $\mathrm{AF}$, or aspirin should be considered in case of contraindications to anticoagulation therapy. 


\section{Conclusion - Up-to-date prevention of thyrotoxic cardiomyopathy and future prospects}

LVH used to be considered reversible in TCMP patients as it was mentioned above. In basic animal research thyroxine induced $\mathrm{LVH}$ was found to be completely reversible when euthyroidism was achieved. Nixon et al. (1979) and Ching et al.(1996) demonstrated LVMI reduction after euthyroidism restoration in patients with thyrotoxicosis. However, it was not proven in more prolonged studies when thyroid function was normalized by thymasol therapy (Merillon et al.1989, Makaruk et al., 1998), According to Marcisz et al (2006) cardiac function and output, as well as systolic blood pressure were altered even after euthyroid state was restored, compared to healthy control group. It could result from TH-induced expression of the genes of contractile proteins and $\beta$-adrenoreceptors. Reversible changes might not be complete and would take longer time.

Beta-adrenergic effects have been investigated for years. Some authors confirmed betablocker benefits for LVH prevention in animal models, while others did not. A number of articles have been recently published regarding the impact of rennin-angiotensin system on $\mathrm{LVH}$ development and potential role of angiotensin converting enzyme inhibitors (ACEi) in $\mathrm{LVH}$ prevention and treatment in thyrotoxicosis. However, all available data are provided by experimental studies. As far as LVH is a common indication for ACEi we studied their effects in humans with TCMP. We found non-complete, but more pronounced reverse changes of LVH and left atrial dilation in patients treated by ACEi (Babenko et al. 2006).

Therefore, recent studies demonstrated high risk of irreversible LVH and left atrial dilation in AF in prolonged thyrotoxicosis and/or in elderly patients. The risk is high even after euthyroidism restoration by antithyroid medication. Beta-blockers and ACEi contribute to a better reversibility of the alterations, but complete recovery is not observed. Therefore, nonreversible (non-medication) treatment seems to be beneficial in a high-risk patients (males, women over the age of 44 years, systolic hypertension) regarding TCMP prevention.

Delayed diagnosis of thyrotoxicosis is of great medical importance as TCMP may manifest at first examination. These patients should be considered for non-medication treatment immediately after euthyroidism is restored.

\section{References}

Agner T. et al. (1984). A reevaluation of atrial fibrillation in thyrotoxicosis. Dan Med Bull 1984, 31:157-159.

Al-Abadi A. (2001). Subclinical thyrotoxicosis. Postgrad Med J . 2001;77:29

Aras D, et al (2005) Simple electrocardiographic markers for the prediction of paroxysmal atrial fibrillation in hyperthyroidism. Int J Cardiol 99:59-64

Armigliato M. et al. (2006). Hyperthyroidism as a cause of pulmonary arterial hypertension: a prospective study. Angiology. 2006 Oct-Nov;57(5):600-6.

Auer J. et al. (2001) Subclinical hyperthyroidism as a risk factor for atrial fibrillation. Am Heart J 2001, 142:838-842

Babenko A. et al. 2006. «Effect of ingibitors converting enzime on echocardiography parametrs at a thyrotoxicosis» poster of 31 annual meting ETA, Italy, Napoly, 26.09 . 
Babenko A. (2006). «Influence of subclinical thyrotoxicosis of varius genesis on cardiovascular system». "Scientific notes of S-Petersburg Medical Uneversity named after Pavlov » 2006, V.13, №4, p.65-69.

Babenko A. (2007) «Remodeling of cardiovascular system at thyreotoxicosis, its influense on vital prognosis» «Arterial hypertension» 2007 V.13, № 1 p.14-21.

Babenko A. (2008) «Character of geometry at clinical and subclinical thyrotoxicosis» «Vestnik of S-Petersburg Uneversity» s. 11 «Medicine», Vol. 4, dec. 2008 г., p.40-46.

Babenko A. et al. (2011). Analisis of contribution of various factors in development and type of remodeling left ventricule at thyreotoxicosis. Bulleten of Almazov Federal Heart, Blood and Endocrinology Centre. - 2011. - N 2 (в печати)

Barst RJ \& Loyd JE. (1998). Genetics and immunogenetic aspects of primary pulmonary hypertension Chest. 1998;114;231-236.

Baumgartner-Parzer S.M. et al. (1997). Increase by triiodothyronine of endothelin-1, fibronectin and von Willebrand factor in cultured endothelial cells . J. Endocrinol. 1997. Vol. 154, No. 2. P. 231-239.

Bianco A et al (2002). Biochemistry, Cellular and Molecular Biology, and Physiological Roles of the Iodothyronine Selenodeiodinases. Endocrine Reviews 2002; 23(1): 38-89.

Bielecka-Dabrowa A et al. (2009) The mechanisms of atrial fibrillation in hyperthyroidism Review. Thyroid Research V.2

Biondi B, et al (1998) Clinical case seminar: reentrant atrioventricular nodal tachycardia induced by levothyroxine. J Clin Endocrinol Metab 83:2643-2645

Biondi B. et al. (1999). Effects of chronic subclinical hyperthyroidism from levothyroxine on cardiac morphology and function. Cardiologia. - 1999. Vol. 44. P. 443-449.

Biondi B. et al. (2000). Endogenous subclinical hyperthyroidism affects quality of life and cardiac morphology and function in young and middle-aged patients. J Clin Endocrinol Metab. 2000; 85: 4701-4705.

Biondi B. et al. (2002). Effects of Subclinical Thyroid Dysfunction on the Heart. Ann Intern Med, Dec. 3, 2002; 137(11): 904-914

Biondi B. et al. (2002). Effects of thyroid hormone on cardiac function: the relative importance of heart rate, loading conditions, and myocardial contractility in the regulation of cardiac performance in human hyperthyroidism. J Clin Endocrinol Metab. 2002; 87: 968-974.

Biondi B, Filetti S, Schlumberger M 2005 Thyroid hormone therapy and thyroid cancer: a reassessment. Nat Clin Pract EndocrinolMetab 1:32-40 363.

Biondi B. \& Cooper D. (2008). The clinical significance of subclinical thyroid dysfunction. Endocr Rev 2008;29:76-131.

Bird T et al. The spectrum of thyroid disease in a community: the Whickham survey. 1977. www.feedback@ist-world.org.

Botella-Carretero JI et al. (2004) Chronic thyrotropin-suppressive therapy with levothyroxine and shortterm overt hypothyroidism after thyroxine withdrawal are associated with undesirable cardiovascular effects in patients with differentiated thyroid carcinoma. Endocr Relat Cancer 11:345-356

Brauman A. et al. (1985). Mitral valve prolapse in hyperthyroidism of two different origins. Br Heart J. 1985. Vol. 53. - P.374-377.

Brent G (1994). The molecular basis of thyroid hormone action. N Engl J Med 1994, 331:847853 
Burggraaf J. et al. (2001) Sympathovagal imbalance in hyperthyroidism. Am J Physiol Endocrinol Metab. 2001. 281: P190-195.

Büssemaker E. et al. (2003). Hyperthyroidism enhances endothelium-dependent relaxation in the rat renal artery. Cardiovascular Research. 2003. Vol.59. P.181-188.

Cacciatori V. et al. (1996). Power spectral analysis of heart rate in hyperthyroidism. J Clin Endocrinol Metab. 1996; 81: 2828-2835.

Canaris G et al. (2000). The Colorado thyroid disease prevalence study. Arch Intern Med. 2000;160:526-34.

Canani LH, et al (2005) The Type 2 Deiodinase A-G (Thr92Ala) Polymorphism Is Associated with Decreased Enzyme Velocity and Increased Insulin Resistance in Patients with Type 2 Diabetes Mellitus. . J Clin Endocrinol Metab. 2005; 90(6): 3472-3478.

Cappola A. et al. (2006). Thyroid Status, Cardiovascular Risk, and Mortality in Older Adults. JAMA. 2006. Vol. 295. P.1033-1041

Chen $\mathrm{Y}$ et al. (2002). Effects of thyroid hormone on the arrhythmogenic activity of pulmonary vein cardiomyocytes. J Am Coll Cardiol 2002, 39:366-372

Ching G. et al. (1996). Cardiac hypertrophy as a result of long-term thyroxine therapy and thyrotoxicosis. Heart. 1996.Vol.75. P. 363-368.

Chu J. et al. (2002). FCCP High Prevalence of Autoimmune Thyroid Disease in Pulmonary Arterial Hypertension. Chest. 2002;122:1668-1673

Coban E. et al. (2006) Endothelial dysfunction in subjects with subclinical hyperthyroidism. J Endocrinol Invest 29:197-200

Cucuianu M. et al. (1987). High levels of plasma von Willebrand factor in hyperthyroidism. Rev Roum Med 25:205-210

Dillmann W. (1990). Biochemical basis of thyroid hormone action in the heart. Am J Med. 1990. 88:626-630

Donatelli M. et al. (2003). Cardiac changes in subclinical and overt hyperthyroid women: retrospective study. Int J Cardiol. 2003. Vol. 90. P.:159-164.

Dorr M. et al. (2001). Low serum thyrotropin is associated with high plasma fibrinogen. Thyroid. 2001. Vol. 11. P. 153-160.

Dorr M. et al. (2005). The association of thyroid function with cardiac mass and left ventricular hypertrophy. J Clin Endocrinol Metab. 2005; 90: 673-677.

Dougherty M \& Craige E. (1973). Apathetic hyperthyroidism presenting as tricuspid regurgitation. Chest 1973;63:767-72.

Dunn M. et al. (1989). Antithrombotic therapy in atrial fibrillation. Chest. 1989;95(2 Suppl):118S-127S.

Erem C (2006) Blood coagulation, fibrinolytic activity and lipid profile in subclinical thyroid disease: subclinical hyperthyroidism increases plasma factor $\mathrm{X}$ activity. Clin Endocrinol (Oxf) 64:323-329

Evangelopoulou M. et al. (1999). Mitral valve prolapse in autoimmune thyroid disease: an index of systemic autoimmunity? Thyroid. 1999. Vol. 9. P. 973-977

Everts $\mathrm{M}$ et al. (1996). Uptake of thyroid hormone in neonatal rat cardiac myocytes. Endocrinology 1996, 137:4235-4242

Faber J et al. (2001). Haemodynamic changes following treatment of subclinical and overt hyperthyroidism. Eur J Endocrinol 2001;145:391-6

Fazio S. et al. 2004. Effects of Thyroid Hormone on the Cardiovascular System. Recent Progr Horm Res 2004, 59:31-50 
Figge J et al. (1994). The clinical evaluation of patients with subclinical hyperthyroidism and free triiodothyronine (free $\mathrm{T}_{3}$ ) toxicosis. Am J Med 1994;96:229-234

Forfar J. et al. (1982). Abnormal left ventricular function in hyperthyroidism: evidence for a possible reversible cardiomyopathy. N Engl J Med. 1982. Vol. 307. P.1165-1175.

Freedberg A et al. (1970). The effect of altered thyroid state on atrial intracellular potentials. J Physiol 1970, 207:357-369

Frost L. et al. (2004). Hyperthyroidism and risk of atrial fibrillation or flutter: a populationbased study. Arch.Intern.Med. 2004. Vol.164. P.1675-1678.

Ganong W (1982). Thyroid hormones and renin secretion. Life Science. 1982. Vol.30. P.577584.

Gerdes A et al. (1985). Failure of propranolol to prevent chronic hyperthyroid induced cardiac hypertrophy and multifocal cellular necrosis in the rat. Can J Cardiol. 1985 Sep-Oct;1(5):340-5.

Goland S. et al. (1999) Dilated cardiomyopathy in thyrotoxicosis. Heart 1999;81:444-445.

Golber L \& Kandror V 1972. Thyreotoxic Heart. Moscow, Russia.

Golf $S$ et al. (1985). Beta-adrenoceptor density and relative number of beta-adrenoceptor subtypes in biopsies from human right atrial, left ventricular, and right ventricular myocard. Cardiovasc Res 19:636-641.

Grineva E. (2009) Type 2 deiodinase Thr92Ala polymorphism impact on clinical course and myocardial remodeling in patients with Graves' disease. Cell Cycle. Vol.8. Is.16 P.2565-2570.

Hoogendoorn E et al. (2004). Subclinical hyperthyroidism: to treat or not to treat? Postgraduate Medical Journal 2004;80:394-398

Homoncik M. et al. (2007) Altered platelet plug formation in hyperthyroidism and hypothyroidism. J. Clin. Endocrinol.Metab. 2007. Vol. 92, №. 8. P. 3006-3012.

Horne $\mathrm{M}$ et al. (2004). Is thyroid hormone suppression therapy prothrombotic? J Clin Endocrinol Metab 200489 4469-4473

Hrnciar J. (2002). Cor thyreotoxicum. Part I - new findings about its etiopathogenesis and diagnosis. Overview of the problem based on 35 years' experience. Vntr Lek. 2002. Vol. 48(1). P. 38-43.

Ikram H. (1977). Haemodynamic effects of beta-adrenergic blockade in hyperthyroid patients with and without heart failure. BMJ 1977;i:1505-7.

Ishikawa T. et al. (1989). Thyroid hormones directly interact with vascular smooth muscle strips. Molecular Pharmaceutics. 1989. Vol.35. P.760-765.

Iwasaki T. et al. (1989) Echocardiographic studies on the relationship between atrial fibrillation and atrial enlargement in patients with hyperthyroidism of Graves' disease. Cardiology. 1989. Vol.76. N 1. P.10-17.

Jiménez E. et al. (1982). Effects of hyper-and hypothyroidism on the basal levels of angiotensin I and kinetic parameters of renin angiotensin system in male rats. Revista Española de Fisiologia. 1982. Vol.38. P.149-154.

Kahaly G. (1987). Graves' disease and mitral valve prolapse. JAMA. 1987. Vol.257.-P. 22-28.

Kim D \& Smith T (1984). Effect of thyroid hormone on sodium pump sites, sodium content, and contractile response to cardiac glycosides in cultured chick ventricular cells. $J$ Clin Invest 1984, 74:1481-1488 
Kiss E et al. (1994). Thyroid hormone induced alterations in phospholamban protein expression. Regulatory effects on sarcoplasmic reticulum calcium transport and myocardial relaxation. Circ Res 75:245-251.

Klein I. (1988). Thyroxine-induced cardiac hypertrophy: time course of development and inhibition by propranolol. Endocrinology. 1988. № 123. P.203-210.

Klein I. (1990). Thyroid hormone and the cardiovascular system. Am. J. of Medicine. 1990. № 88. P.631-637.

Klein I \& Levey GS. (2000). The cardiovascular system in thyrotoxicosis. In: Braverman LE, Utiger RD, eds. The thyroid, 8th ed. Philadelphia: Lippincott-Raven, 2000;596-604.

Klein I. \& Ojamaa K. (2001). Thyroid hormone and cardiovascular system. N Engl J Med, 2001Vol. 344, No. 7 February 15, p.501-509.

Kobori $\mathrm{H}$ et al. (1999). Local renin-angiotensin system contributes to hyperthyroidisminduced cardiac hypertrophy. J. Endocrinol. 1999. V.160, I. 1, 43-47.

Kohrle J. Local activation and inactivation of thyroid hormones: the deiodinase family. Mol Cell Endocrinol. 1999 May 25; 151(1-2):103-19.

Ladenson P. (1993). Thyrotoxicosis and the heart: something old and something new[Editorial]. J Clin Endocrinol Metab. 1993. Vol. 77. P. 332-333.

Levina L. (1989). Heart at endocrine diseases. Leningrad, Russia.

Lombardi G. et al. (1994). Heart and Thyroid. Wien. - P.86-91.

Lutton S. et al. (2001) Cardiomyopathy and myocardial failure.

Maia A et al (2005) Type 2 iodothyronine deiodinase is the major source of plasma T3 in euthyroid humans. J.Clin. Invest. 2005; 115: 2524-2533

Makaruk B et al. (1998). Changes of echocardiographic indices of the left cardiac ventricle systolic function during thyrostatic therapy. Polish J Endocrinol, 1998; 49: 307-12.

Manger J et al. (1988). Congestive heart failure and sudden death in a young woman with thyrotoxicosis. West J Med 1988; 149:86-91

Marchant C.et al. (1993). Renin-angiotensin system in thyroid dysfunction in rats. J.of Cardiovascular Pharmacology. - 1993. - Vol.22. - P.449-455.

Marcisz C et al.( 2001). Influence of short-time application of a low sodium diet on blood pressure in patients with hyperthyroidism or hypothyroidism during therapy. Am J Hypertens. 2001; 14: 995-1002

Marcisz C. et al. (2006). Left ventricular mass in patients with hyperthyroidism. Med Sci Monit. 2006. 12(11) P.481-486.

Marqusee E et al. (1998). Subclinical thyrotoxicosis. Endocrinol Metab Clin North Am. 1998;27:37-49

Martí V et al. (1997). Myocardial damage does not occur in untreated hyperthyroidism unless associated with congestive heart failure. Am Heart J. 1997 Dec;134(6):1133-7

Menzel T et al. (2000). Pathophysiology of impaired right and left ventricular function in chronic embolic pulmonary hypertension: changes after pulmonary thromboendarterectomy. Chest 118: 897-903, 2000.

Mercé J. et al. (2005). Cardiovascular abnormalities in hyperthyroidism: A prospective Doppler echocardiographic study. Am. J.Medicine (2005) 118, 126-131.

Merillon J et al. (1981). Left ventricular function and hyperthyroidism. Br Heart J, 1981; 46: 137-43.

Metso, S. et al. (2007) Increased Cardiovascular and Cancer Mortality after Radioiodine Treatment for Hyperthyroidism J Clin Endocrinol Metab 2007;92:2190-2196 
Mier A et al (1989) Reversible respiratory muscle weakness in hyperthyroidism. Am Rev Respir Dis 139,529-533

Nakazawa H et al. (2000). Is there a place for the late cardioversion of atrial fibrillation? A long-term follow-up study of patients with post-thyrotoxic atrial fibrillation. Eur Heart J. 2000; 21: 327-333.

Nakchbandi I et al. (1999) Pulmonary Hypertension Caused by Graves' Thyrotoxicosis* Normal Pulmonary Hemodynamics Restored by 131I Treatment Chest. 1999;116:1483-1485.

Napoli R et al. (2001) Impact of Hyperthyroidism and Its Correction on Vascular Reactivity in Humans. Circulation. 2001;104:3076

Nordyke R et al. (1988) Graves' disease: influence of age on clinical findings. Arch Intern Med. 1988; 148: 626-631.

Nixon J et al. (1979). Alterations in left ventricular mass and performance in patients treated effectively for hyrotoxicosis. A comparative echocardiographic study. Am J Med. 1979 Aug;67(2):268-76 .

Ojamaa K et al. (1999) Regulation of rat cardiac Kv1.5 gene expression by thyroid hormone is rapid and chamber specific. Endocrinology. 1999.140:3170-3176.

Okura H \& Takatsu Y. (1994) High-Output Heart Failure as a Cause of Pulmonary Hypertension. Intern. Med. Vol. 33, No. 6 June 1994

Ortmann C et al. (1999) Inflammation of the cardiac conduction system in a case of hyperthyroidism. Int J. Legal. Med. 1999. № 112. - P.271-274.

Osman F. et al. (2000) Cardiac rhythm abnormalities in thyrotoxicosis - the explanation for excess vascular mortality J.Endocrinology. - 2000. - Vol.164. -P.321.

Osman F. et al. (2002). Cardiac Dysrhythmias and Thyroid Dysfunction - The Hidden Menace? J Clin Endocrinol Metab. 2002 Vol. 87, No. 3 963-967.

Osman F. et al. (2007) Cardiovascular Manifestations of Hyperthyroidism Before and After Antithyroid Therapy. J Am Coll Cardiol, 2007; 49:71-81

Pachucki J, et al (2001) Type 2 Iodothyronine Deiodinase Transgene Expression in the Mouse Heart Causes Cardiac-Specific Thyrotoxicosis. Endocrinology. 2001; 142(1): 13-20.

Palmieri E et al. (2004) Myocardial contractility and total arterial stiffness in patients with overt hyperthyroidism: acute effects of beta1-adrenergic blockade. Eur J Endocrinol. 2004; 150: 757-762.

Paran Y et al. (2006) Pulmonary hypertension and predominant right heart failure in thyrotoxicosis. Resuscitation. 2006; 69: 339-341.

Parle J. et al.(2001). A single low serum thyrotrophin (TSH) concentration predicts increased all-cause and cardiovascular mortality in older persons in the community: a 10-year cohort study. Lancet. 2001. Vol.358. P.861-865.

Parle J. et al. (1991). Prevalence and follow-up of abnormal thyrotrophin (TSH) concentrations in the elderly in the United Kingdom. Clin Endocrinol (Oxf). 1991. № 34. P.77-83.

Peeters R et al (2003). Polymorphisms in Thyroid Hormone Pathway Genes Are Associated with Plasma TSH and Iodothyronine Levels in Healthy Subjects? J Clin Endocrinol Metab. 2003; 88(6): 2880-2888. 
Peeters R et al. (2006). Genetic variation in thyroid hormone pathway genes; polymorphisms in the TSH receptor and the iodothyronine deiodinases. Eur. J. Endocrinol. 2006; 155(5): 655-662.

Petersen P. \& Hansen J.M. (1988) Stroke in thyrotoxicosis with atrial fibrillation. Stroke. 1988. Vol.19. P.15-18.

Pilo A et al. (1990) Thyroidal and peripheral production of 3,5,3'-triiodothyronine in humans by multicompartmental analysis. Am J Physiol 1990; 258:715-726

Polikar R. et al. The thyroid and the heart. Circulation. 1993.Vol. 87. P.1435-1441.

Presti C. \& Hart R. Thyrotoxicosis, atrial fibrillation, and embolism, revisited. Am.Heart J. 1989. Vol.117. P.976-977.

Report of the 1995 World Health Organization/International Society and Federation of Cardiology Task Force on the Definition and Classification of Cardiomyopathies. Circulation. 1996. Vol. 93. P. 841-842

Roffi M. et al. (2003) Thyrotoxicosis and the cardiovascular system: Subtle but serious effects. Cleveland Clinic Journal Of Medicine. 2003. Vol. 70.P. 57-63.

Ross D (1996) Subclinical thyrotoxicosis. In: Braverman LE, Utiger RD, eds. Werner and Ingbar's The Thyroid: A Fundamental and Clinical Text. 7th ed. Philadelphia: Lippincott Williams \& Wilkins; 1996:1016-20.

Rubin L. \& Badesch D. (2006) Thyrotoxicosis as a Risk Factor for Pulmonary Arterial Hypertension. Ann Intern Med, February 7, 2006; 144(3): 222 - 223.

Salvatore D, et al (1996). Type 2 iodothyronine deiodinase is highly expressed in human thyroid. J Clin Invest 1996; 98:962-968.

Samuels M (1998) Subclinical thyroid disease in the elderly. Thyroid.1998;8:803-13

Sandier G. \& Wilson G. (1959)The nature and prognosis of heart disease in thyrotoxicosis: a review of 150 patients treated with I131. QJM 1959. Vol. 28. P. 347-352.

Sawin C. T. et al.(1994) Low serum thyrotropin concentrations as a risk factor for atrial fibrillation in older persons. N Engl J Med. 1994. Vol. 331.-P. 1249-1254.

Scivoletto R. et al. (1986) Thyroid hormones and vascular reactivity: role of the endothelial cell. Eur. J. Pharm. 1986. Vol.129. P.271-278.

Shapiro L et al. (1997) Minimal cardiac effects in asymptomatic athyreotic patients chronically treated with thyrotropin-suppressive doses of 1-thyroxine. J Clin Endocrinol Metab. 1997;82:2592-5.

Sgarbi J et al (2010) Subclinical thyroid dysfunctions are independent risk factors for mortality in a 7.5-year follow-up: the Japanese-Brazilian thyroid study.Eur J Endocrinol 2010;162:569-77.

Siu C-W. et al. (2007) Incidence, clinical characteristics and outcome of congestive heart failure as the initial presentation in patients with primary hyperthyroidism. Heart 2007;93:483-487.

Soh M \& Croxson M. (2008) Fatal thyrotoxic cardiomyopathy in a young man. BMJ. 2008; 337: a531

Squizzato A. et al. (2007) Thyroid Dysfunction and Effects on Coagulation and Fibrinolysis: A Systematic Review. J Clin Endocrinol Metab.2007. Vol. 92, No. 7 2415-2420

Staffurt J et al. (1977) Arterial embolism in thyrotoxicosis with atrial fibrillation. $\mathrm{Br} \mathrm{Med} \mathrm{J}$ $1977,2: 688-690$

Toft A \& Boon N (2000) Thyroid disease and the heart. Heart 2000;84:455-460 
Yanai-Landau H et al (1995) Autoimmune aspects of primary pulmonary hypertension. Pathobiology. 1995;63(2):71-5.

Yung C. et al. (2006) Hemodynamic Changes in Hyperthyroidism-Related Pulmonary Hypertension: A Prospective Echocardiographic Study. J Clin Endocrinol Metab. 2006 Vol. 92, No. 5 1736-1742

Vargas F et al. (2006) Vascular and renal function in experimental thyroid disorders. Eur. J. Endocrinol., 2006, Vol 154, I 2, 197-212.

Wang C \& Crapo L (1997)The epidemiology of thyroid disease and implications for screening. [PMID: 9074859] Endocrinol Metab Clin North Am. 1997;26:189-218

Whitner T. et al. (2005) Hyperthyroidism. Presenting as Isolated Tricuspid Regurgitation and Right Heart Failure. Tex Heart Inst J. 2005; 32(2): 244-245.

Woeber K (1992) Thyrotoxicosis and the heart. N Engl J Med 1992;327:94-98.

Xenopoulos NP et al.(1996) Severe right heart failure in a patient with Graves' disease. Clin Cardiol 1996;19:903-5. 


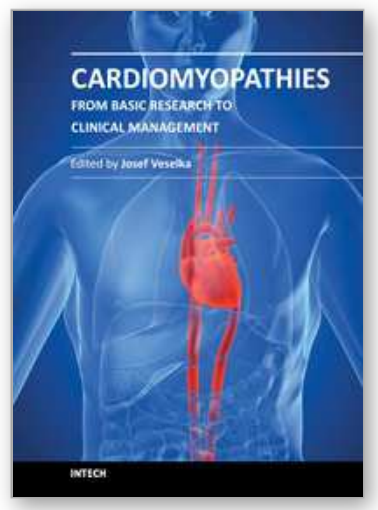

\author{
Cardiomyopathies - From Basic Research to Clinical Management \\ Edited by Prof. Josef Veselka
}

ISBN 978-953-307-834-2

Hard cover, 800 pages

Publisher InTech

Published online 15, February, 2012

Published in print edition February, 2012

Cardiomyopathy means "heart (cardio) muscle (myo) disease (pathy)". Currently, cardiomyopathies are defined as myocardial disorders in which the heart muscle is structurally and/or functionally abnormal in the absence of a coronary artery disease, hypertension, valvular heart disease or congenital heart disease sufficient to cause the observed myocardial abnormalities. This book provides a comprehensive, state-of-theart review of the current knowledge of cardiomyopathies. Instead of following the classic interdisciplinary division, the entire cardiovascular system is presented as a functional unity, and the contributors explore pathophysiological mechanisms from different perspectives, including genetics, molecular biology, electrophysiology, invasive and non-invasive cardiology, imaging methods and surgery. In order to provide a balanced medical view, this book was edited by a clinical cardiologist.

\title{
How to reference
}

In order to correctly reference this scholarly work, feel free to copy and paste the following:

Alina Yu. Babenko, Alekber A. Bairamov, Elena N. Grineva and Eugenia O. Ulupova (2012). Thyrotoxic Cardiomyopathy, Cardiomyopathies - From Basic Research to Clinical Management, Prof. Josef Veselka (Ed.), ISBN: 978-953-307-834-2, InTech, Available from: http://www.intechopen.com/books/cardiomyopathies-frombasic-research-to-clinical-management/cardiomyopathy-at-ddysfunction-of-thyroid

\section{INTECH}

open science | open minds

\author{
InTech Europe \\ University Campus STeP Ri \\ Slavka Krautzeka 83/A \\ 51000 Rijeka, Croatia \\ Phone: +385 (51) 770447 \\ Fax: +385 (51) 686166 \\ www.intechopen.com
}

\author{
InTech China \\ Unit 405, Office Block, Hotel Equatorial Shanghai \\ No.65, Yan An Road (West), Shanghai, 200040, China \\ 中国上海市延安西路65号上海国际贵都大饭店办公楼 405 单元 \\ Phone: +86-21-62489820 \\ Fax: +86-21-62489821
}


(C) 2012 The Author(s). Licensee IntechOpen. This is an open access article distributed under the terms of the Creative Commons Attribution 3.0 License, which permits unrestricted use, distribution, and reproduction in any medium, provided the original work is properly cited. 